Archived version from NCDOCKS Institutional Repository http://libres.uncg.edu/ir/asu/

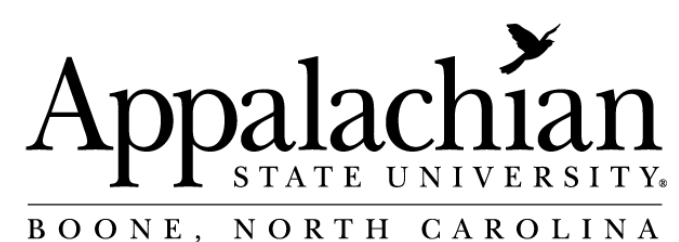

\title{
If We Build It, They Might Come: An Empirical Investigation of Supply and Demand in the Recruitment of Rural Psychologists
}

By: John Paul Jameson, Michael B. Blank, and Dianne L. Chambless

\begin{abstract}
Past research has established that practicing doctoral level psychologists tend to cluster in metropolitan areas, leaving the rural population gravely underserved. Discussions of this problem have assumed that psychologists hold negative attitudes toward rural work for many reasons, leading to a supply shortage. The present study attempts to examine the accuracy of this hypothesis by examining attitudes of current doctoral students in clinical psychology. Eight hundred eighty-four students responded to an online survey. Results indicate that attitudes toward rural practice were not overwhelmingly negative as predicted. Additionally, a significant number of respondents endorsed positive attitudes toward rural practice. A follow-up study was conducted to determine if the shortage of rural psychologists might be attributed to a lack of demand from rural employers. A search of three popular job resources revealed that few rural jobs were advertised, indicating weak demand for psychologists in rural areas. Results indicate that efforts to recruit psychologists to rural areas should be rethought, and increasing the visibility of available opportunities for psychologists should be made a priority.
\end{abstract}

John Paul Jameson, Michael B. Blank, and Dianne L. Chambless (2009) "If We Build It, They Might Come: An Empirical Investigation of Supply and Demand in the Recruitment of Rural Psychologists" Journal of Clinical Psychology, Vol. 65(7), pp. 723-735 Version of Record Available From (onlinelibrary.wiley.com) 
If We Build It, They Might Come: An Empirical Investigation of Supply and Demand in the Recruitment of Rural Psychologists

Though prevalence rates of mental illnesses differ little between rural and urban areas (Kessler et al., 1994), individuals in rural areas are often faced with barriers to access to specialty mental health services. The problems of establishing an adequate mental health service delivery system in rural areas are numerous and complex, and solutions will likely need to be multifaceted (see Jameson \& Blank, 2007, for a review). Among the most pressing mental health needs of rural areas is to ameliorate the shortage of specialty mental health service providers (Bird, Dempsey, \& Hartley, 
2001; Merwin, Goldsmith, \& Manderscheid, 1995; Murray \& Keller, 1991). In 1999, $87 \%$ of federally designated Mental Health Professional Shortage Areas were located in nonmetropolitan areas (Bird et al., 2001). The most rural counties in the United States are 4.7 times more likely than metropolitan counties to have a shortage of mental health professionals (Merwin, Hinton, Dembling, \& Stern, 2003). Furthermore, because the Community Mental Health Centers Act of 1963 established catchment areas centered in urban hubs (Blank, Fox, Hargrove, \& Turner, 1995), rural areas are less likely to have community mental health centers (Merwin, Snyder, \& Katz, 2006). This evidence is particularly alarming considering the apparent direct relationship between the supply of specialty mental health providers and mental health service utilization (Hauenstein et al., 2006; Lambert \& Agger, 1995).

Although the notion that clinical psychologists can single-handedly solve rural America's mental health service problems seems absurd, we have asserted that they represent a crucial part of the solution because of the unique skill set they possess (Jameson \& Blank, 2007). In addition to direct provision of services, psychologists often possess skills in program development, consultation, evaluation and research, teaching, policy development, and advocacy that are invaluable in the effort to improve rural mental health services. The actual number of psychologists practicing in rural areas is difficult to determine because no national database exists to locate all practicing psychologists. However, a study of the distribution of clinical psychologists using data from the Health Resources and Service Administration suggests a paucity of rural psychologists similar to that of other mental health professionals nationwide (Merwin et al., 2003). Thus, it is presumed that rural areas suffer severe shortages of psychologists, and efforts should be made to increase their numbers.

Hargrove (1991) proposed several hypotheses to explain why rural service may be unattractive to clinical psychologists: (a) graduates are not equipped with the generalist skills necessary to practice effectively in rural areas because of increased specialization in clinical psychology programs; (b) clinical psychologists often move quickly into administrative or supervisory roles in rural service organizations, an unattractive prospect for those wanting to practice; (c) rural mental health services are dominated by the public sector, and both starting salaries and earning potential for positions in these organizations cannot compete with those offered by private organizations in urban areas; and (d) rural areas do not offer the activities and services that psychologists grow accustomed to during graduate training.

We propose several additional reasons why rural practice might be unappealing to clinical psychologists. First, we believe that many individuals perceive rural areas as having limited cultural diversity. Although this is not necessarily true, the perception may limit the desirability of rural practice. Second, we believe psychologists may perceive vast differences between their social values and those held by many people in rural areas. This differentiation can be viewed as an opposition between liberalleaning psychologists (Redding, 2001) and the values of the conservative red states that contain most of the rural population. Third, rural practice may be viewed negatively because of fear of burnout and professional isolation (Roberts, Battaglia, \& Epstein, 1999). Research suggests that this fear may not be unfounded: Kee, Johnson, and Hunt (2002) found that rural practitioners suffer very high rates of burnout and that burnout was predicted by a lack of integration with other professionals. Fourth, we believe that psychologists may view rural practice negatively because of lifestyle and reasons surrounding family and community. 
Particularly, educational opportunities for children and chances for finding romantic partners with similar educational and ideological backgrounds may be viewed as more limited in rural areas. Finally, psychologists may not be drawn to rural areas because they do not believe jobs exist or they are too difficult to find.

There are many positive aspects of rural psychology practice that have not been widely discussed, and these may play an important role in efforts to bring clinical psychologists to rural areas. The enhanced personal aspects of living and working in a rural environment may be accentuated to reduce negative attitudes among psychologists. Rural areas can often be characterized by an intimate sense of community and a more relaxed pace of life with comparatively little crime, pollution, and traffic and abundant recreational activities. These factors can make rural service more alluring. Furthermore, the relative autonomy in clinical practice and the challenge of working with a wide variety of cases might be seen as attractive to many psychologists.

The shortage of psychologists in rural areas has been discussed primarily as an issue of supply. That is, the assumption seems to exist that ample opportunities are available for psychologists in rural areas, but psychologists are not willing to fill these roles. Programs such as the National Health Service Corps Loan Repayment Program (Department of Health and Human Services, National Health Services Corps, 2008), which offers repayment of educational loans in return for work in underserved areas, reflect this assumption.

However, the alternative explanation that there is little demand for rural psychologists has not been widely explored. For the purpose of the current discussion, demand refers to market demand generated from agencies that may potentially hire psychologists (i.e., the presence of unfilled positions for psychologists). This can be contrasted to need, which reflects the requirements of the population for psychological services. Clearly, the mental health needs of rural inhabitants have not met by specialty mental health providers, including psychologists (Blank, Fox, Hargrove, \& Turner, 1995; Fox, Merwin, \& Blank, 1995; Gamm, Stone, \& Pittman, 2003; Goldsmith, Wagenfeld, Manderscheid, \& Stiles, 1997; Murray \& Keller, 1991). However, it is not known if demand for psychologists matches the need.

An understanding of basic market forces is imperative to designing initiatives to bring more psychologists to rural practice. If the demand for psychologists outstrips the supply, then efforts should be focused on promoting positive aspects of and eliminating negative myths about rural practice. Conversely, if the demand for rural psychologists is weaker than the supply of willing practitioners, more emphasis should be placed on promoting the unique skill set offered by psychologists to rural mental health service systems.

The current studies attempted to explore the willingness of psychologists to work in rural areas as well as to examine the demand for their services in rural areas. The objective of Study 1 was threefold. First, we wished to examine whether attitudes toward rural practice among future psychologists were indeed largely negative. Second, we tested the hypotheses that (a) individuals who have grown up in rural areas have more positive attitudes toward rural practice than individuals from nonrural areas, (b) students in graduate programs that offer training experiences in rural areas will have more positive attitudes, and (c) students enrolled in programs located in rural areas will view rural practice more positively. Finally, we wished to examine empirically the hypotheses proposed by Hargrove and those by the present authors to identify perceptions that contribute to negative attitudes toward rural 
practice, as well as to identify any perceived positive aspects of rural practice and living that could be used to inform recruitment efforts. The objective of Study 2 was to gauge the demand for doctoral level psychologists in rural areas by examining the location of job postings in media sources popular among advanced doctoral students. We considered the proportion of rural jobs in the listings to serve as a metric of demand for clinical psychologists in rural areas.

\section{Study 1: Attitudes toward Rural Practice}

\section{Method}

Participants. We decided to direct the survey to current doctoral students for several reasons. First, doctoral students are likely to be searching for a position in the near future. They represent the psychology workforce of the immediate future, and will likely fill a large proportion of open clinical positions. Second, they are likely to be the most mobile. Because their career has not yet started, it is less likely that they are tied to a particular geographic region or organization. Third, doctoral students are easily accessed in large numbers through their training organizations, and are generally amenable to completing online surveys.

Directors of clinical training of doctoral training programs in clinical psychology were asked to distribute an invitation to a Web-based questionnaire to current students via e-mail. Solicited programs included those with membership in the National Council of Schools and Programs in Professional Psychology (NCSPP) as well as those with clinical directors with membership in the Council of University Directors in Clinical Psychology (CUDCP). In the case of NCSPPmember programs, clinical directors were contacted using the organization's listserv; the investigators contacted clinical directors with membership in CUDCP individually. The invitation described the survey as an exploration of attitudes toward working in various environments. No explicit mention of rural work was made in the invitation. Representatives from a total of 239 clinical psychology programs were solicited (158 CUCP programs, 77 NCSPP programs, 4 programs with membership in both organizations). Of these, six programs contacted the first author and explicitly refused to distribute the invitation; 1,037 students responded. Ninety-nine students reported no interest in clinical work after graduation, and were therefore excluded from the analysis. Five respondents were also excluded because they indicated that they were seeking a terminal master's degree. Further, 49 students were excluded because of nonresponse to over $33 \%$ of the questions, leaving 884 respondents in the analysis. The total number of students currently enrolled in the represented programs who received the invitation to participate could not be enumerated, and therefore, a response rate could not be calculated.

Measures. Information was collected regarding the type of degree sought (PsyD or $\mathrm{PhD}$ ), current year of study, and desired career after graduation (e.g., research/ teaching, private practice, hospital practice). Respondents were also asked to describe the population of the location they grew up in. Respondents who reported growing up in a town of less than 20,000 people were considered to have a rural background; individuals reporting growing up in a town or city with a population greater than 20,000 were considered to have a nonrural background. Information regarding the location of their graduate program and the availability of opportunities for training in rural areas was also collected. 
The Attitudes toward Rural Practice Survey (ARPS) questionnaire, developed by the authors for this study, featured two sections: the General Attitudes section (ARPS-GA) and the Specific Perceptions section (ARPS-SP). Both sections consisted of statements that were rated on a 5-point Likert-type scale $(1=$ strongly disagree; $2=$ disagree; $3=$ neither agree nor disagree; $4=$ agree $; 5=$ strongly agree). The ARPS-GA is a 4-item measure designed to assess general attitudes toward rural practice: (a) whether the respondent would consider working in a rural area if quality jobs were available, (b) whether the respondent believed rural practice would be a rewarding experience, (c) whether the respondent had a favorable view of living in a rural area, and (d) whether the respondent believed it likely that he or she would work in a rural area in the future. Possible scores range from 5 to 20, with higher scores indicating more positive attitudes. The ARPS-GA showed adequate internal consistency in the sample $(\alpha=.84)$.

The ARPS-SP is intended to measure perceptions toward specific personal and professional aspects of rural practice. Individual items were derived directly from hypotheses proposed by Hargrove (1991) as well as the authors' review of the literature. The measure consists of two subscales: the positive aspects subscale, which composed of statements describing potential benefits of rural practice (e.g., "Rural practice is appealing to me because the pace of rural life is less stressful"; "Rural practice is appealing to me because I will have the opportunity to see a wide variety of cases"); and the negative aspects subscale, which consists of statements describing potential drawbacks (e.g., "Rural practice is unappealing to me because I would have limited contact with people who share my social values"; "I would not want to practice in a rural area because the caseloads are too heavy"). Internal consistency was adequate for both the 7-item positive aspects subscale $(\alpha=.83)$ and the 17-item negative aspects subscale $(\alpha=.86)$. Because the scales differ in length, item means are used throughout (range $=1-5$ ). Higher item means indicated higher endorsement of items on the scale (i.e., higher scores on the negative aspects subscale indicate more negative perceptions; higher scores on the positive aspects subscale indicates more positive perceptions).

Procedure. All procedures and materials were approved by the University of Pennsylvania Institutional Review Board. Respondents completed an online survey hosted by Surveymonkey.com, a Web-based business that provides hosting and technical services for online questionnaires. Because the detailed ARPS-SP items could potentially influence responses on the ARPS-GA, participants completed the ARPS-GA prior to completing the ARPS-SP. The order of items on both sections was randomized for each participant to prevent order effects.

\section{Results and Discussion}

Descriptive data are presented in Table 1. As anticipated, the majority of respondents reported growing up in nonrural areas and were currently enrolled in programs located in nonrural areas, supporting the notion that psychology is indeed an urban profession. Surprisingly, a substantial proportion of respondents reported that their program offers training in a rural setting.

Data from the ARPS-GA were examined to determine whether attitudes toward rural practice were largely negative among doctoral students. The distribution of item means is presented in Figure 1. Taken with the distribution, the item mean $(M=3.04, S D=.88, N=876)$ suggests that the distribution centers around a neutral attitude towards rural practice, with most respondents having slightly negative or 
Table 1

Attitudes Toward Rural Practice Survey-Specific Perceptions section (ARPS-SP) Subscale and Item Means and Standard Deviations

\begin{tabular}{lrr}
\hline & $M$ & $S D$ \\
\hline ARPS-SP Negative aspects & 2.93 & 0.56 \\
Lack activities and services & 3.69 & 1.15 \\
Limited opportunities to interact with other professionals & 3.43 & 1.05 \\
Limited continuing education opportunities & 3.26 & 1.00 \\
Limited educational opportunities to my children & 3.24 & 1.15 \\
Lack of cultural diversity & 3.23 & 1.20 \\
Limited contact with people who share my social values & 3.18 & 1.24 \\
Little potential for salary growth & 3.10 & 0.91 \\
Starting salaries are lower than in nonrural areas & 3.09 & 0.95 \\
Limited opportunity to see patients in my specialty & 3.02 & 1.07 \\
Few good jobs available & 2.90 & 0.95 \\
Jobs are too hard to find & 2.86 & .913 \\
Limited patient contact/administrative duties & 2.71 & 0.81 \\
Would be unable to find a romantic partner & 2.60 & 1.21 \\
Caseloads are too heavy & 2.48 & 0.75 \\
Forced to work in the public sector & 2.38 & 0.81 \\
Would not be able to establish a private practice & 2.38 & 0.82 \\
Wide variety of patients is unappealing & 2.27 & 0.85 \\
ARPS-SP Positive aspects & 3.03 & 0.70 \\
Safe for family & 3.27 & 0.94 \\
Pace of life less stressful & 3.22 & 1.10 \\
Intimate sense of community & 2.99 & 1.06 \\
Opportunity to see a wide variety of cases & 2.98 & 0.98 \\
Greater personal autonomy & 2.94 & 0.96 \\
Greater professional autonomy & 2.89 & 0.91 \\
Excellent recreational opportunities & 2.89 & 1.04 \\
\hline
\end{tabular}

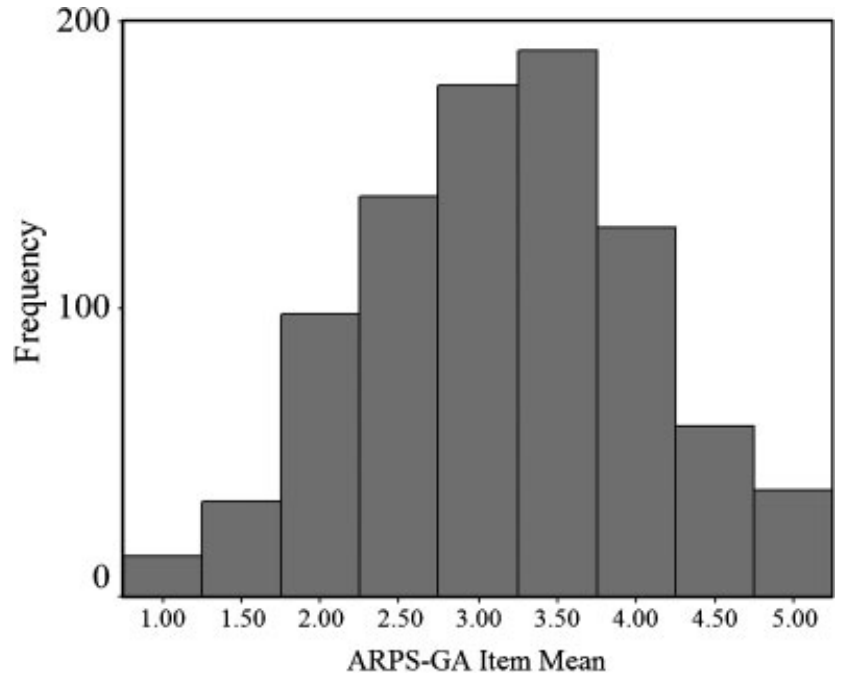

Figure 1. Distribution of attitudes toward Rural Practice Survey-General Attitudes section (ARPS-GA) responses. 
slightly positive attitudes. The small skewness index for the distribution of the ARPS-GA data $(S k=-.017, S E=.09)$ also provides evidence that respondents do not hold overwhelmingly negative attitudes toward rural practice. Instead, attitudes may be better described as roughly normally distributed.

Similar results were found for both the ARPS-SP positive aspects $(M=3.03$, $S D=.70, N=839)$ and negative aspects $(M=2.93, S D=.56, N=839)$ subscale item means. Distributions of the subscale item means are presented in Figure 2. As with results from the ARPS-GA, skewness indices are low (ARPS-SP positive aspects: $S k=-.22, S E=.08$; ARPS-SP negative aspects: $S k=-.33, S E=.08$ ). Results suggest that individuals soon to enter the psychology job market do not see rural practice as possessing overwhelming negative qualities or completely lacking in positive qualities. Perceptions of specific positive and negative aspects of rural practice approximate normal distributions.

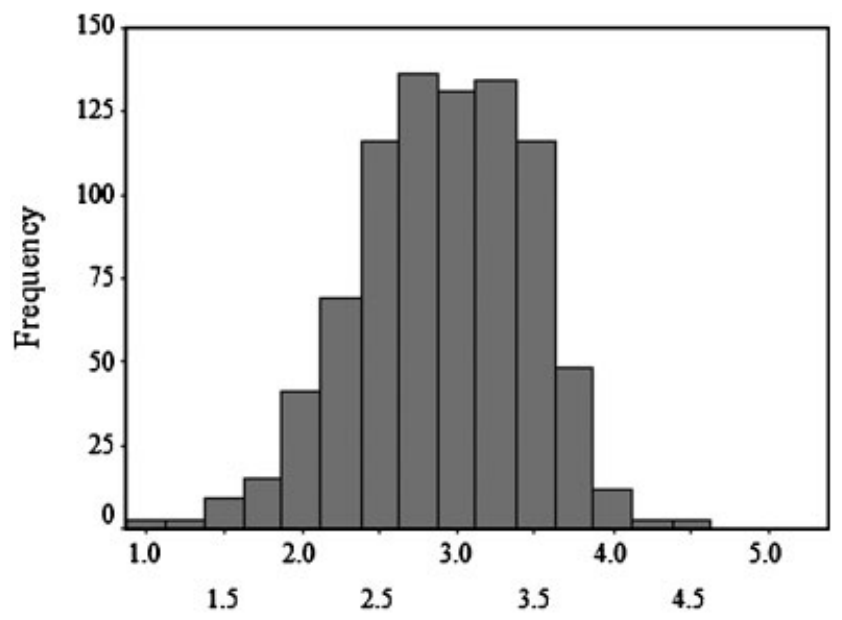

ARPS-SA Negative subscale item mean

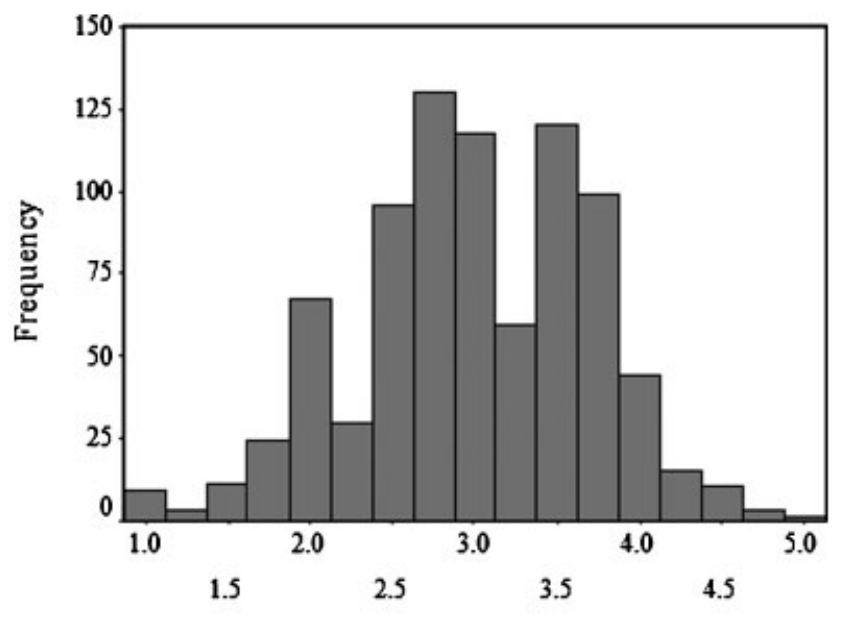

ARPS-SA Positive subscale item mean

Figure 2. Distributions of ATtitudes toward Rural Practice Survey- Specific Perceptions section (ARPSSP) subscales. 
Simultaneous linear regression was employed to determine whether positive perceptions and negative perceptions uniquely predicted general attitudes toward rural practice. Both the ARPS-SP positive aspects subscale, $\beta=.54, t(831)=22.91$, $p<.001, r_{\text {part }}=.48$; and negative aspects subscale, $\beta=.38, t(831)=16.32, p<.001$, $r_{\text {part }}=-.34$; significantly predicted scores on the ARPS-GA, with part correlations indicating that the subscales made unique contributions to the model. These data suggest that attitudes toward rural practice are not simply a product of overwhelming negative perceptions about the quality of personal and professional life in rural areas. Positive perceptions also seem to play a significant role in determining general attitudes. Based on this finding, we urge that efforts to recruit early-career psychologists to rural areas focus not only on dispelling negative ideas regarding work and life in rural areas, but also on highlighting the many positive aspects.

Mean responses for individual ARPS-SP items are presented in Table 2. Both the ARPS-SA negative aspects and positive aspects subscale distributions center around neutral perceptions of the factors. Only one item (Rural practice is unappealing to me because rural communities lack the activities and services I am accustomed to.) had a mean greater than one standard deviation above the subscale mean, lending partial support to the hypothesis proposed by Hargrove (1991). However, other factors proposed by Hargrove (1991) and the present authors did not receive substantial support from these data. The relatively restricted range of the item means suggests that multiple factors influence the attitudes of early career psychologists toward rural practice, or that the most important factors are highly idiosyncratic.

$t$ Tests were used to determine the relationship between general attitudes toward rural practice and rural upbringing, the availability of rural training opportunities, and graduate program location. Results indicate that individuals from rural areas had higher item means on the ARPS-GA than individuals from nonrural areas, $t(874)=10.57, p<.001, d=0.8$, indicating more positive attitudes. Additionally, individuals enrolled in programs offering rural training opportunities had higher ARPS-GA scores than those in programs without rural training, $t(687)=5.07$, $p<.001, d=0.39$. ARPS-GA scores did not differ significantly between students enrolled in rural or nonrural programs, $t(874)=1.24, p=.21, d=.13$. We note that students who grew up in rural areas are more likely to attend programs located in rural areas, $\chi^{2}(1, n=884)=4.06, p<.05$, OR $=1.55$, and programs that offer rural training, $\chi^{2}(1, n=694)=4.33, p<.05$, OR $=1.42$, but do not favor these programs

Table 2

Employment Resource Choices Provided to Respondents $(N=385)$

Resource

Weighted rank sum score

APA Monitor/PsycCareers ${ }^{\mathrm{a}}$

State government job listings ${ }^{\mathrm{a}}$

Local government job listings

Monster.com $^{\mathrm{a}}$

Careerbuilder.com

\footnotetext{
${ }^{\mathrm{a}}$ Resources included in the analysis.
} 
overwhelmingly. In fact, 204 of the $242(84 \%)$ respondents from rural areas were enrolled in programs located in nonrural areas. These data suggest that efforts to recruit new psychologists for rural service should not necessarily focus on graduates of rural programs. Instead, psychologists native to rural areas are likely to be most attracted to rural positions, regardless of the location of their program.

Taken as a whole, these data do not support the notion that there is a lack of early career psychologists willing to serve in rural areas. Opinions toward rural practice among doctoral psychology students soon to enter the workforce were not overwhelmingly negative. On the contrary, $35.9 \%$ of respondents in the survey had mildly, moderately, or strongly positive attitudes toward working and living in rural areas as measured by the ARPS-GA.

We note several weaknesses in Study 1. First, the study examined hypotheses related to perceptions of practicing and living in rural areas, not the reality of living and practicing in rural areas. These perceptions may be based on stereotypes of rural areas, and there is no evidence to suggest that they accurately reflect the experiences of practitioners actually working in rural areas. Therefore, one may reasonably question the validity of the study. Second, the attitudes of doctoral students may not accurately reflect the attitudes of licensed psychologists who are ready to join the workforce. The respondents' lack of experience may have caused them to underestimate the impact of negative factors, or idealize the positive factors. Further, a majority the respondents appear to have little experience with rural areas, and may have insufficient information to make judgments about the appeal of rural practice. Third, positive attitudes toward rural practice do not directly translate accepting a position in a rural area in the future. The endorsement of items on a survey does not have the gravity that making a long-term commitment to live and work in a sparsely populated area does. Further, these data do not demonstrate that a significant number of individuals would prefer a rural position to an identical one located in an urban or suburban area. Future research endeavors should focus on addressing these shortcomings.

Study 2: The Availability of Rural Job Opportunities for Psychologists

\section{Method}

Selection of sources. Participants in Study 1 were asked to choose and rank three employment resources that they would most likely use when beginning to search for their first job after graduation from a list of 12 free electronic resources. Respondents in their first, second, or third year of graduate study were excluded for this study, as more advanced students were thought to be more likely to have given extensive thought to their plans for employment. Three hundred fifty-eight advanced students completed this section of the survey. Because we were concerned primarily with clinical practice careers, resources featuring teaching and research positions only (e.g., Chronicle of Higher Education) were not included among the options. Resources received a score of 3 each time they were ranked as a first choice, a score of 2 each time they were ranked as a second choice, and a score of 1 for each third choice ranking. These weighted scores were summed, and the three most popular were searched. The complete list of available resource choices and their weighted scores are provided in the Table 2.

Though ranked third by respondents, we decided to omit local government listings for two reasons. First, we believe that few rural organizations would advertise in local government media because of the lack of qualified candidates in rural areas. 
For this reason, a search of local government resources would likely heavily favor urban areas. Second, a representative sample of job listings from the total number of listings in the United States could not be conceived. Rural areas are not as likely to have easily accessible local government job listings that are up-to-date. Therefore, sampling frames would likely favor urban locales as well. For these reasons, we searched APA Monitor/PsycCareers, state government listings, and Monster.com.

Search strategies. All searches were conducted via the Internet. To maximize the number of relevant positions captured in the search, search engines were not used. Instead, all listings were searched manually under all healthcare and social services categories. In cases where state Websites did not employ a job categorization scheme, all postings were searched. Multiple positions under a single listing were counted separately. Eight state sites did not have positions for psychologists posted at the time of the search. Further, two state sites did not provide necessary information on availability or job locations. We attempted to contact the employment offices in these states to obtain the necessary information, but our requests were denied. Therefore, the analysis includes job listings from 40 states.

Job listings were included in the analyses if they required a minimum of master's degree and specifically mentioned doctoral level candidates or required full licensure. Further, job descriptions had to include at least one of the following duties: (a) direct patient contact, (b) supervision or training of staff providing services to consumers, or (c) the development of treatment protocols or policy for the provision of services. Case management and care coordination positions were not included in the analyses.

Rural/nonrural definitions. Two definitions of rurality were used in the analyses. In the first analysis, job positions were classified as rural or nonrural based on the United States Department of Agriculture Rural Urban Continuum Codes (United States Department of Agriculture Economic Research Service, 2003). Jobs located in counties with an urban population of less than 20,000 (corresponding with codes 7 , 8, and 9) were considered rural, whereas jobs located in counties with urban populations of 20,000 or more were considered nonrural. This represents a stringent definition of rural, in that these counties contain only approximately $5 \%$ of the U.S. population.

A second analysis was conducted using much more liberal definitions of rural and nonrural: the United States Census Bureau's definitions of metropolitan and nonmetropolitan counties. Under these definitions, a county is considered metropolitan if it contains all or part of a centralized, densely populated area of 100,000 or more residents. All counties not meeting these criteria are considered nonmetropolitan, and contain approximately $20 \%$ of the U.S. population.

\section{Results and Discussion}

The proportion of open positions located in rural areas was relatively low across each of the three resources searched. One hundred sixty-nine positions that met criteria were found through the APA Monitor/PsycCareers at the time of the search. Of these, only $6(3.6 \%)$ were located in rural counties. A search of state sites yielded 290 open positions, $18(6.2 \%)$ of which were located in a rural county. Finally, of the 107 eligible positions found on Monster.com, only $3(2.8 \%)$ were located in rural counties.

The analysis was repeated with listings for positions at prisons and forensic hospitals removed. A disproportionate number of prisons are located in rural areas 
(Beale, 2003). It is likely that psychologists employed at these institutions provide services to inmates and staff exclusively. Therefore, inclusion of these positions may not accurately reflect demand for psychologists providing services to rural residents.

After removing forensic positions from APA Monitor/PsycCareers job listings, 149 positions remained in the analysis. Only three $(2.0 \%)$ of these positions were located in rural counties. Similarly, only 1 nonforensic position of the 104 positions $(0.96 \%)$ found on Monster.com was located in a rural county. State listings featured a slightly higher proportion of rural positions: Eight of the 138 nonforensic positions $(5.7 \%)$ were located in rural counties.

The second set of analyses classified the same job positions as metropolitan or nonmetropolitan based on United States Census Bureau definitions. Of the 169 APA Monitor/PsycCareers listings, $29(17.2 \%)$ were located in nonmetropolitan counties. Excluding forensic positions, 20 of the 149 listings $(13.4 \%)$ were located in nonmetropolitan counties. Eight of the 107 total positions $(7.5 \%)$ and 6 of the 104 nonforensic positions found on Monster.com were located in nonmetropolitan counties. Positions in nonmetropolitan counties were better represented on state Websites. Ninety-four of the 290 total positions and 52 of the 138 nonforensic positions $(37.7 \%)$ were located in nonmetropolitan counties.

Results suggest that rural areas are not well represented among open positions posted in popular job search resources, especially when forensic positions are excluded. Further, the analyses suggest that the most rural areas contain the fewest opportunities for psychologists. Only in the state listings did the proportion of rural jobs approach or exceed the proportion of the population living in rural areas. Considering that a shortage of psychologists and other mental health professionals already exists in these areas, these data paint a relatively grim picture for the future of the rural psychology workforce.

Results of the analysis can be interpreted in one of two ways. Optimistically, the data suggest that advertising methods employed by rural mental health service organizations do not effectively target early-career psychologists. There may exist alternative methods that rural organizations use widely for recruiting, such as local advertising or word-of-mouth recruiting. Limited resources may prevent rural organizations from advertising in the more popular resources. Given the overwhelming favoritism shown towards APA Monitor/PsycCareers job listings, these alternative methods may not be reaching a large number of qualified candidates. Such tactics may simply redistribute psychologists already in rural practice and do little to attract greater numbers of new practitioners to rural areas.

An alternative explanation for these data is that rural positions for psychologists simply do not exist in large numbers. This is likely due to the lack of resources in rural mental health services systems. Funding may be insufficient to employ doctoral level clinicians, who command higher salaries than bachelor or master's level clinicians. Rural counties have been shown to spend less per capita on mental health services than nonrural areas (Rohland \& Rohrer, 1998).

There are several limitations to the current study. First, the use of responses from advanced graduate students to select the resources that were examined may be problematic. Many of these respondents likely have not started searching for their first job, and may not be familiar with the resources available. Individuals with prior experience searching for jobs may have ranked the resources differently, providing a more accurate picture of the job market. A second shortcoming of the study is the fact that the resources were searched only once each, during the winter and spring of 2007. Seasonal patterns of the job market may have influenced the results. 


\section{General Conclusions}

Results from the two studies suggest that the shortage of psychologists in rural areas may be best characterized as a demand problem rather than a supply problem. These findings run counter to assumption that a short supply of candidates is responsible for the absence of rural psychologists (Hargrove, 1991; Hargrove \& Breazeale, 1993; Murray \& Keller, 1991). The attitudes of psychologists-in-training indicate that a significant proportion see rural practice as a rewarding experience. However, the lack of open positions in rural areas is indicative of a barren job market for psychologists. The findings suggest that current strategies for placing psychologists in rural areas should be reconsidered. Instead of relying solely on doctoral programs specializing in rural mental health to attract psychologists to rural areas, more focus should be placed on creating positions with competitive salaries and benefits and advertising these positions effectively. Further, recruiting efforts should take place in the national marketplace. An overwhelming number of trainees report searching for job listings in national resources. Though initiatives such as the National Health Service Corps Loan Repayment Program represent an important strategy for rural recruitment, they may be overlooked by individuals searching for jobs. Offering agencies additional funding for the advertisement of these positions in the national marketplace may make such programs more effective for relatively little additional cost.

\section{References}

Beale, C.L. (2003). Rural prisons: An update. Rural Development Perspectives, 11, 25-27.

Bird, D., Dempsey, P., \& Hartley, D. 2001. Addressing mental health workforce needs in underserved rural areas: Accomplishments and challenges. Portland, ME: Edmund S. Muskie School of Public Service, Maine Rural Health Research Center.

Blank, M.B., Fox, J.C., Hargrove, D.S., \& Turner, J.T. (1995). Critical issues in reforming rural mental health service delivery. Community Mental Health Journal, 31, 511-524.

Department of Health and Human Services, National Health Services Corps. (2008). Join Us: Loan Repayment Program. Retrieved April 17, 2008, from http://nhsc.bhpr.hrsa.gov/ join_us/lrp.asp

Fox, J., Merwin, E., \& Blank, M. (1995). De facto mental health services in the rural south. Journal of Health Care for the Poor and Underserved, 6, 434-469.

Gamm, L., Stone, S., \& Pittman, S. (2003). Mental health and mental disorders - a rural challenge: A literature review. In L.D. Gamm, L.L. Hutchison, B.J. Dabney, \& A.M. Dorsey (Eds), Rural healthy people 2010: A companion document to Rural Healthy People 2010 (Vol. 2, pp. 97-113). College Station, TX: The Texas A\&M University System Health Science Center, School of Rural Public Health, Southwest Rural Health Research Center.

Goldsmith, H.F., Wagenfeld, M.O., Manderscheid, R.W., \& Stiles, D. (1997). Specialty mental health services in metropolitan and nonmetropolitan areas: 1983 and 1990. Administration \& Policy in Mental Health, 24, 475-488.

Hauenstein, E.J., Petterson, S., Merwin, E., Rovnyak, V., Heise, B., \& Wagner, D. (2006). Rurality, gender, and mental health treatment. Family \& Community Health, 29, 169-185.

Hargrove, D.S. (1991). Training Ph.D. psychologists for rural service: A report from Nebraska. Community Mental Health Journal, 27, 293-298.

Hargrove, D.S., \& Breazeale, R.L. (1993). Psychologists and rural services: Addressing a new agenda. Professional Psychology: Research and Practice, 24, 319-324. 
Jameson, J.P., \& Blank, M.B. (2007). The role of clinical psychology in rural mental health services: Defining problems and developing solutions. Clinical Psychology: Science and Practice, 14, 283-298.

Kee, J.A., Johnson, D., \& Hunt, P. (2002). Burnout and social support in rural mental health counselors [electronic version]. Journal of Rural Community Psychology, E5. Retrieved May 21, 2008, from http://www.marshall.edu/jrcp/sp2002/Kee.htm

Kessler, R.C., McGonagle, K.A., Zhao, S., Nelson, C.B., Hughes, M., Eshleman, S., et al. (1994). Lifetime and 12-month prevalence of DSM-III-R psychiatric disorders in the United States. Results from the National Comorbidity Survey. Archives of General Psychiatry, 51, 8-19.

Lambert, D., \& Agger, M.S. (1995). Access of rural AFDC Medicaid beneficiaries to mental health services. Health Care Financing Review, 17, 133-145.

Merwin, E.I., Goldsmith, H.F., \& Manderscheid, R.W. (1995). Human resource issues in rural mental health services. Community Mental Health Journal, 31, 525-537.

Merwin, E., Hinton, I., Dembling, B., \& Stern, S. (2003). Shortages of rural mental health professionals. Archives of Psychiatric Nursing, 17, 42-51.

Merwin, E., Snyder, A., \& Katz, E. (2006). Differential access to quality rural healthcare: Professional and policy challenges. Family \& Community Health, 29, 186-194.

Murray, J.D., \& Keller, P.A. (1991). Psychology and rural America: Current status and future directions. American Psychologist, 46, 220-231.

Redding, R.E. (2001). Sociopolitical diversity in psychology: The case for pluralism. American Psychologist, 56(3), 205-215.

Roberts, L.W., Battaglia, J., \& Epstein, R.S. (1999). Frontier ethics: Mental health care needs and ethical dilemmas in rural communities. Psychiatric Services, 50, 497-503.

Rohland, B.M., \& Rohrer, J.E. (1998). County funding of mental health services in a rural state. Psychiatric Services, 49, 691-693.

United States Department of Agriculture Economic Research Service. (2003). Measuring rurality: Rural-urban continuum codes. Retrieved November 11, 2004, from http:// www.ers.usda.gov/briefing/rurality/RuralUrbCon/ 